$\xi=$ 圈

\title{
Enhancement of fingerprint image using wiener filter
}

\author{
R. Devi ${ }^{1 *}$, P. Sujatha ${ }^{2}$ \\ ${ }^{1}$ Asst. Professor, Department of Information Technology, Vels University, Chennai. Research Scholar, \\ Bharathiar University, Coimbatore \\ ${ }^{2}$ Asso. Professor, Department of MCA, A.C Patil College of Engineering, Kharghar, Navi Mumbai \\ *Corresponding author E-mail: devi.scs@velsuniv.ac.in
}

\begin{abstract}
A fingerprint is one of the most vital Biometric traits used for Personal Identification. To identify and match the fingerprint accurately, it has to be enhanced efficiently. In this paper, an efficient fingerprint enhancement technique is adopted and compared with the existing methods. The proposed methodology consists of three Phases. In the first phase, the fingerprint is subjected to the de-noising process. After adding noise such as salt and pepper, Gaussian and speckle noise, the image is blurred. In the second phase, the fingerprint is filtered with Wiener filter and then de-blurred. In the third, the filtered image is further enhanced for more clarity. The paper emphasizes, the fingerprint preprocessing followed with the enhancement produces better quality image. The performance of the proposed methodology is compared and evaluated using two performances measures namely Peak-Signal-Noise -Ratio and Mean Squared Error using Matlab R2013a.
\end{abstract}

Keywords: Otsu Thresholding Binarization; Histogram Equalization; Wiener Filter; Gaussian Noise; Salt and Pepper; Speckle.

\section{Introduction}

A tremendous growth in the advances of information technology evenly requires security for personal identification and authentication. It leads to the automatic personal identification systems on Biometrics. The Biometric system provides a reliable solution to the problem of identifying an individual by acquiring the physiological or behavioral characteristics such as fingerprint, iris, palm print, hand geometry, voice, signature, etc. Biometric features play an imperative role for personal identification. Among them, fingerprint place a significant role and have a high level of reliability. Fingerprint recognition is one of the primary tasks of the Integrated Automated Fingerprint Identification Service (IAFIS).[1] it contains fingerprint classification, fingerprint enhancement, and fingerprint matching. A fingerprint is referred as an outline of interleaved ridges and valleys on the tip of the finger. It is an impression left by the friction ridges in which each ridge is defined by one pixel wide. The different forms of ridges, where it emerges, ends, splits or merges with another ridge is termed as minutiae. There are two types of minutiae namely ridge ending and bifurcation. The number of incidence of minutiae differs from one fingerprint to another. The fingerprint image has to be enhanced and further pre-processed to skeletonize the ridges and to make them clear, to identify the minutiae correctly. The minutiae are extracted, and the value of the friction ridges is manipulated for fingerprint verification and matching. A numerous automatic minutiae extraction algorithm have been proposed and are carried out by the researchers. An important aspect of every research done in image processing must require a reliable and standard input image.[1] In practice, the clamor might adulterate the fingerprint. This defilement may prompt the awful print and decrease the effectiveness of the outcome. Therefore, fingerprint enhancements techniques are essential and ought to be performed to improvise an image before processing. The paper mainly focuses on the possible enhance- ment steps to refine the fingerprint into a better quality image. Most of the authors enhances the fingerprint through some morphological operations and finally filter the noisy image using existing filters. This paper includes all the possible morphological operation for enhancing the fingerprint before and after filtering. To work out a distinct and effective result the proposed methodology involves Otsu thersholding Binarization, Histogram equalization, adding noise , blurring then deblurring and filtering using Wiener filter. The filtered fingerprint is further enhanced using morphological operations such as contrast and sharpening and thinning.

\section{Literature survey}

P.Gnanasivam et al. proposed two stages of processing for fingerprint image enhancement. In the first stage, the fingerprint is enhanced using Gabor filter and the second stage involves refinement of ridges using three stages Morphological filtering followed by the thinning process. Bilinear interpolation is done to resize the image into the standard size. The image is first normalized and then segmented followed by the Morphological filtering transformation. The enhancement step involves normalization, segmentation and filtering. [2]

Neethu et al. uses Fourier transform algorithm, and is possibly done by multiplying the FFT of the block by its magnitude a set number of times. The algorithm manipulates the ridges finely thicker and skeletonize. The author also states that if the magnitude scale of the FFT is squared, power or cubed, an image will be still better.The enhancement step involves contrast, histogram equalization, binarzation and then finally filtered using FFT. [1] Subba Reddy Borra et al., proposed methodology using three modules. In the first module the fingerprint is subject to denoising process using Wave Atom transform, then in the second 
module image is enhanced using an optimization algorithm namely Modified Cuckoo search.[3]

Feng Zhao et al. developed a methodology that enhances the fingerprint using adaptive thresholding and thinning followed by minutiae extraction .[4]

Sanghoon Lee et al. developed a method where a color fingerprint image is segmented and thinned using gradient based approach.

Om Preeti Chaurasia developed an algorithm that includes normalization, segmentation and finally image de-noising using Directional Weighted Median filter. The enhancement of involves binarization, central line thinning, dilation, refining followed by filtering.[5]

I.Golda Seelia et al. proposed a methodology that includes histogram equalization for contrast and Wiener filter and Gobar filter for noise reduction. The fingerprint image is enhanced by applying binarization and adaptive thresholding.[6]

Raju Rajkumar and K Hemachandran proposed a Directional filter technique which is one of the multi-resolution enhancement methods. It removes the noise from the fingerprint image by decomposing the image into several frequency bands or sub images.[7] Greenberg et al. proposed a fast fingerprint enhancement algorithm based on an anisotropic filtering. The enhancement involves histogram equalization, wiener filtering, Binarizarion followed by morphological filtering.[8]

\section{Proposed methodology}

The proposed methodology for Fingerprint preprocessing is illustrated below.

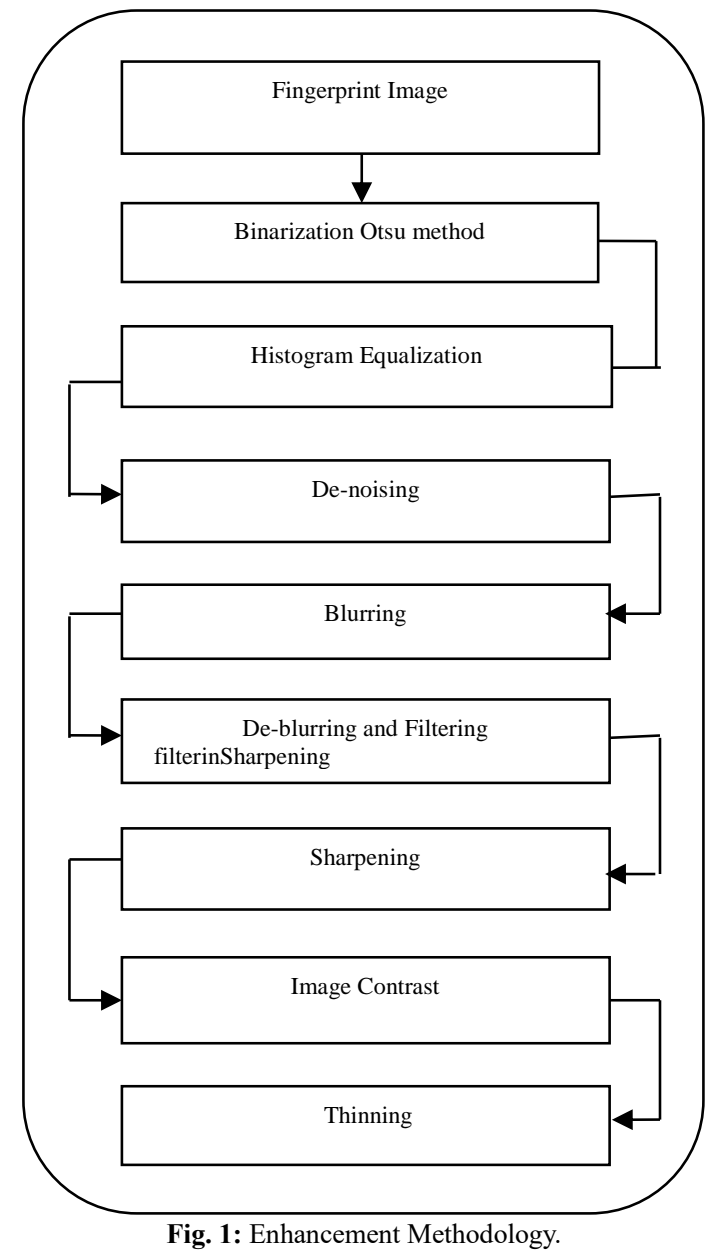

Fig 1 briefly explains the fingerprint pre processing methodology using Weiner filter. To refine the ridges three enrichment operations were adopted.

Step 1: Input fingerprint gray image is first converted into the Binary image using Otsu method.
Step 2: The Binary image is brightened through histogram equalization.

Step 3: The equalized Binary image is de-noised and blurred too.

Step 4: The de-noised and blurred image is filtered using Wiener filter.

Step 5: The filtered image is further contrasted and sharpened Step 6: The enhanced fingerprint image is finally thinned.
(A)

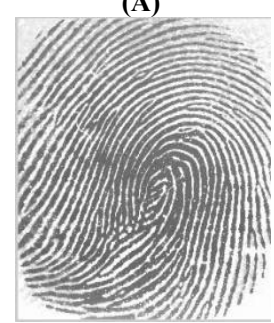

(B)

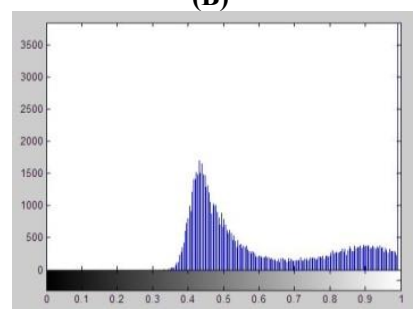

Fig. 2: (A): Original Image Fig. 2(B): Histogram of Original Image

The above Fig2 (a) shows the original image and Fig2 (b) gives the histogram of the given fingerprint image.

\subsection{Binarization using otsu thresholding}

The Binarization is a process of converting a gray image into a double image. The function im $2 \mathrm{bw}$ changes the grayscale image to double image. The binary images are in the form of 0 's and 1's. 0 to represent the black pixels whereas 1 to represent the white pixels. Otsu thresholding technique is used for binarization. The procedure replaces all pixels in the input image with the luminance more prominent than level with the esteem 1 [5].The primary motivation behind the changing of an input image into a binary image is to compute the threshold value. It involves examining the grayscale value of each pixel in an enhanced image. If the pixel values are greater than the threshold value then the pixels are converted to white pixels or else the pixels are converted to black pixels.
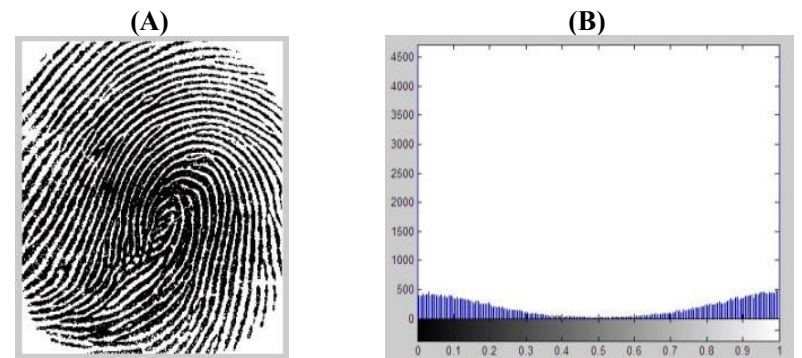

Fig. 3: (A): Binary Image Fig. 3(B): Histogram of Binary Image.

Fig 3(a) Coverts the input gray image into Binary image using Otsu thresholding and Fig3 (b) shows the histogram of the Binary image.

\subsection{Histogram equalization}

The image is further enhanced through histogram equalization. The histogram equalization improves the contrast of images by transforming the values 1 and 0 .The black and white pixels are turned to visualize the picture brighter. The histogram of an image provides the intensity level in the image as a graphical representation.[9].
(A)

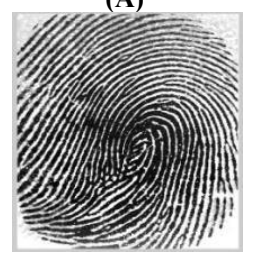

(B)

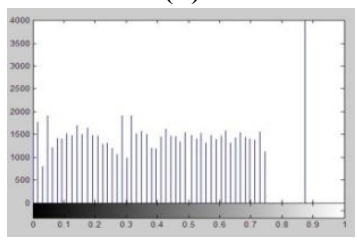

Fig. 4: (A): Histogram Equalization Fig. 4(B): Histogram of Equalized Image. 
Fig 4(a) shows the histogram equalization of a binary image and Fig 4(b) shows the histogram of equalized image.

\subsection{De-noising and blurring}

The Noise removal in images is an important part of image processing. The above image is de-noised using three different noises namely salt and pepper, Gaussian and speckle noise. The noisy fingerprint is then blurred to adapt the filtering technique.

(A)

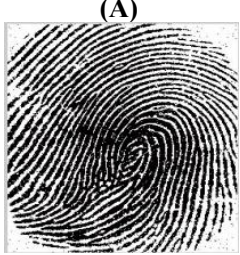

Fig. 5: (A) Noisy Image Fig. 5(B): Blurred Image.

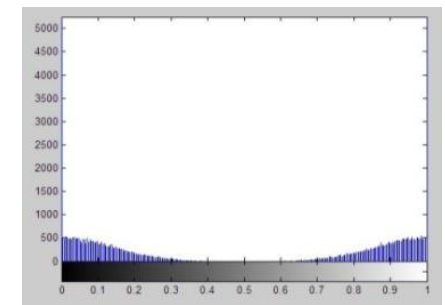

Fig. 5: (C) Histogram of Noisy Image.

The above Fig 5(a) shows the noisy image, specifically salt and pepper noise is added to the image .This noisy image is further blurred given in Fig 5(b). The histogram of noisy image is shown in Fig 5(c).

\subsection{De-blurring and filtering}

Images are often corrupted by random variations in intensity, illumination or have poor contrast. So images are filtered for enhancement and smoothening. Filtering increases the pixel intensity values. In our proposed methodology, the noisy and the blurred images are de-blurred and filtered using Wiener de-blurring filter.
(A)

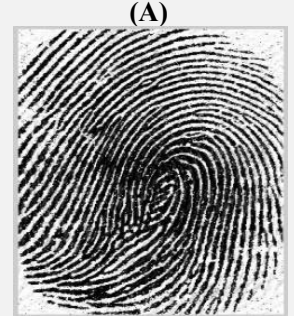

(B)

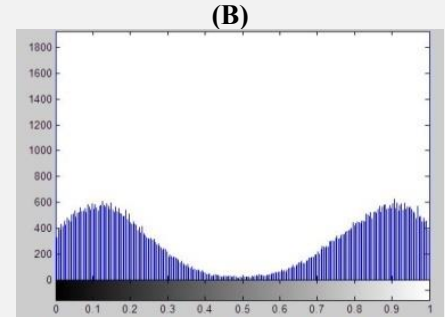

Fig. 6: (A) Filtered Image Fig. 6 (B) Histogram of Filtered Image.
Fig 6(a) shows the filtered image. Wiener filter is one of the most important filtering techniques to remove the noise as well the blurred image. Fig 6(b) shows the histogram of filtered image. To actualize the Wiener filtering, it is basic to compute the power spectra of the original image and the noise. The main aim of this filter is to minimize the mean squared error. Consider the Wiener filtering frequency domain as $\mathrm{W}(\mathrm{u}, \mathrm{v})$. The restored image states as[10].

$\mathrm{Xn}(\mathrm{u}, \mathrm{v})=\mathrm{W}(\mathrm{u}, \mathrm{v}) . \mathrm{Y}(\mathrm{u}, \mathrm{v})-$ Equation 1.[12]

In equation 1 , Where $\mathrm{Y}(\mathrm{u}, \mathrm{v})$ is the received signal, and $\mathrm{Xn}(\mathrm{u}, \mathrm{v})$ is the restored image.

\subsection{Fingerprint enhancement}

The filtered image is further enhanced through two operations namely sharpening and contrast. The input image is first bright- ened through two-step process which includes binarization and histogram equalization. The filtered image is first sharpened and then contrast to increase the intensity of a picture. The function imsharpen () returns an enhanced gray scale image where the image features such as edges has been sharpened. Sharpening increases the contrast along the edges where different colors meet. The contrast function enhances the intensity of an image equally. It makes the image to gaze brighter. Enhancement after filtering increases the intensity of an image to a higher level and also it gives a better image quality.

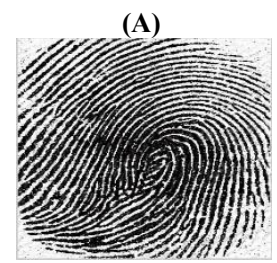

(B)

Fig. 7: (A) Sharpened Image Fig. 7 (B) Contrast Image.

Fig 7(a) and 7(b) shows the enhanced images after filtering.

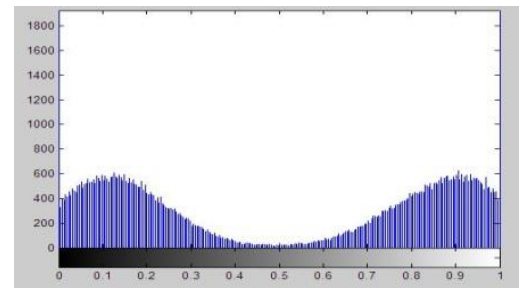

Fig. 7: (C) Histogram of Enhanced Fingerprint

Fig 7(c) shows the enhanced fingerprint image after filtering.

\subsection{Thinning}

After enhancing, the fingerprint is thinned using Central Line Thinning algorithm in which black pixels are converted into white, whereas white pixels are kept as such[5]. There are almost 27 different thinning algorithms, whereas Central Line Thinning algorithm is one of the better algorithms that retain the original image after thinning process. Thinning is one of the morphological operations applied to a binary image. It is used to identify and eliminate the redundant pixels of ridges which are one pixel wide. Thinning is the final enhancement step performed before minutiae extraction. It is an iterative process, and it is repeated continuously until all ridges become one pixel wide. Ridges are thinned for efficient minutiae detection[11].

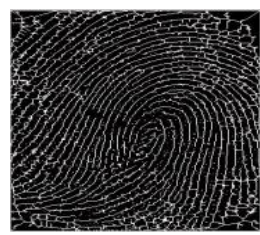

Fig. 8: Thinning Image.

Fig 8 shows the thinning image after enhancement to make the fingerprint clearer.

\section{Results and discussions}

\subsection{Dataset}

The proposed methodology has been implemented in Matlab 2013, and particular Image processing Toolbox has been used for fingerprint filtering and enhancement [3]. The implementation has been done on the fingerprints obtained from FAC2000, consist of four databases namely DB1, DB2, DB3, and DB4. Each database is acquired through different sensor devices. In this paper, DB2 is 
used. The fingerprints obtained by using a low-cost capacitive sensor and the image size is $256 \times 364$. The database consists of two sets namely set A comprises of 800 fingerprints and set B comprises of 100 fingerprints with the resolution of $512 \mathrm{dpi}$. The fingerprints in set B were trained and tested for results. The samples chosen for the performance were of .tiff format.

\subsection{Performance measures}

Image Enhancement improves the quality of a digital image. The techniques that provide better quality image given by the authors vary from person to person. So it is necessary to ascertain some practical measures to compare the enhancement techniques that produce better quality image. The two important metrics to define the quality of the image are Peak-Signal- Noise-Ratio (PSNR) and Mean Squared Error (MSE). PSNR is an expression that calculates the ratio of the maximum possible value of a signal and the power of distorting noise that affects the image quality. It is commonly represented in decibel value. The Mathematical notation of PSNR is

$\left.\operatorname{PSNR}=20^{*} \log (\max (\max (\mathrm{f}))) /(\mathrm{MSE})^{\wedge} 0.5\right)-$ Equation 2

Equation 2 calculates the PSNR value of the given image using the formula, Where $f$ represents the original Image, max is the maximum signal value that exists for a good image.

The MSE represents the average errors between the actual image and the noisy image. The error is the quantity by which the values of the original image differ from the noisy image. The mathematical notation of MSE is

$\left.\operatorname{MSE}=\left(1 / m^{*} n\right)\right)^{*} \operatorname{sum}\left(\operatorname{sum}\left((\mathrm{f}-\mathrm{g}) .^{\wedge} 2\right)\right)-$ Equation 3

Equation 3 .calculates the MSE value of the given image using the above formula., where $f$ represents the original image, $g$ accounts for the noisy image, $m$ represents the number of rows of pixels of an image, $n$ represents the number of columns of pixels of the image and Max is the maximum signal value that exists in our original image.

\subsection{Experimental observation}

The parameters measures like Peak Signal Noise Ratio (PSNR) and Mean Squared Error (MSE) values for the images, before and after enhancing are tabulated and also represented in the form of graphical representation. The effectiveness of the proposed technique is analyzed by adding different types of noises to the fingerprint images and the same images are enhanced using the proposed wiener filtering technique. The noises that are added for testing are Salt and pepper, Gaussian and Speckle. Table 1.gives the PSNR and MSE values for the enhanced fingerprint by adding salt \& pepper noise and Figure $8 \& 9$ gives the graphical representation of the parameter measures separately. Table 2 gives the PSNR and MSE values for the enhanced fingerprint by adding Gaussian noise and Figure $10 \& 11$ shows the graphical representation of the parameter measures separately. Table 3 gives the PSNR and MSE values for the enhanced fingerprint by adding Speckle noise and Figure $12 \& 13$ shows the graphical representation of the parameter values separately.

The Proposed methodology is also compared with the existing methodologies based on the PSNR value. Table 4 shows the comparative analysis and Figure 14 gives the pictorial representation of the comparison. From the above experimental observation the PSNR value of the proposed methodology provides an average of $73 \%$ which seems to be a better value when compared to the existing.

\subsection{Adding salt and pepper noise}

Table 1 signifies the input image that undergone Pre-processing steps which include adding of salt and pepper noise, blurring, filtering, and enhancement. The Table1 also represents the performance measures namely Peak-signal-noise-ratio and MeanSquared-Error after each iteration.

Table 1: Enhanced Fingerprint Images with Its PSNR and MSE

\begin{tabular}{|c|c|c|c|c|c|c|c|c|c|c|c|c|c|}
\hline S.No & $\begin{array}{l}\text { Input } \\
\text { Image }\end{array}$ & $\begin{array}{c}\text { Peak- } \\
\text { Signal- } \\
\text { Noise- } \\
\text { Ratio }\end{array}$ & $\begin{array}{c}\text { Mean } \\
\text { Square } \\
\text { Error }\end{array}$ & $\begin{array}{c}\text { De- } \\
\text { noising }\end{array}$ & $\begin{array}{c}\text { Peak- } \\
\text { Signal- } \\
\text { Noise- } \\
\text { Ratio }\end{array}$ & $\begin{array}{c}\text { Mean } \\
\text { Square } \\
\text { Error }\end{array}$ & Blurring & Filtering & $\begin{array}{c}\text { Peak- } \\
\text { Signal- } \\
\text { Noise- } \\
\text { Ratio }\end{array}$ & $\begin{array}{l}\text { Mean } \\
\text { Squar } \\
\text { e } \\
\text { Error }\end{array}$ & $\begin{array}{l}\text { Enhance } \\
\text { Image }\end{array}$ & $\begin{array}{c}\text { Peak- } \\
\text { Signal- } \\
\text { Noise- } \\
\text { Ratio }\end{array}$ & $\begin{array}{l}\text { Mean } \\
\text { Squa } \\
\text { re } \\
\text { Error }\end{array}$ \\
\hline 1 & & 61.6432 & 0.04 & & 58.4065 & 0.09 & & & 64.9774 & 0.02 & & 70.7693 & 0.02 \\
\hline 2 & & 60.4011 & 0.06 & & 57.8824 & 0.11 & & & 66.2368 & 0.02 & & 72.1761 & 0.01 \\
\hline 3 & & 58.5430 & 0.05 & & 57.4098 & 0.09 & & & 65.0993 & 0.02 & & 74.2848 & 0.01 \\
\hline 4 & & 61.3019 & 0.05 & & 59.2849 & 0.08 & & & 65.7818 & 0.02 & & 70.1464 & 0.02 \\
\hline 5 & & 58.2473 & 0.10 & & 57.1134 & 013 & & & 65.3227 & 0.01 & & 73.3225 & 0.01 \\
\hline
\end{tabular}


(A)

PSNR Of Enhanced Fingerprint

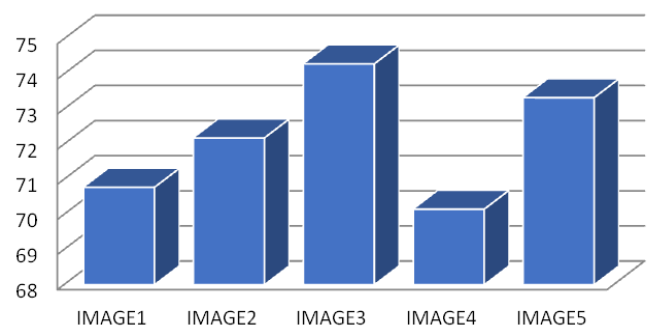

(B)

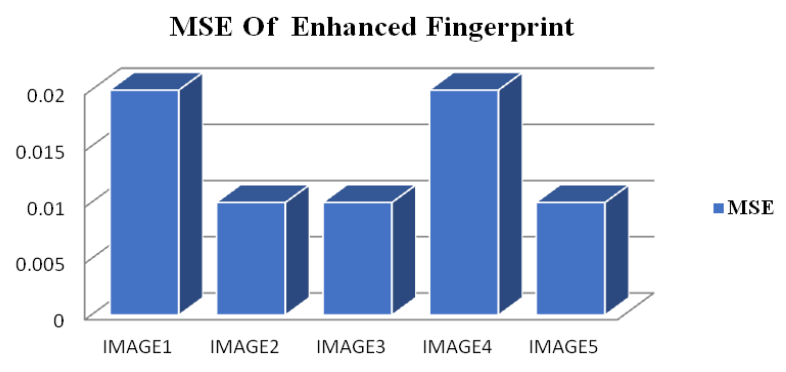

Fig. 9: (A) Graphical Representation for PSNR Values of Enhanced Fingerprint after the Removal of Salt \& Pepper Noise. (B) Graphical Representation for MSE Values of Enhanced Fingerprint after the Removal of Salt \& Pepper Noise.

\subsection{Adding gaussian noise}

Table 2: Enhanced Fingerprint Images with Its PSNR and MSE

\begin{tabular}{|c|c|c|c|c|c|c|c|c|c|c|c|c|c|}
\hline S.No & $\begin{array}{l}\text { Input } \\
\text { Image }\end{array}$ & $\begin{array}{l}\text { Peak- } \\
\text { Signal- } \\
\text { Noise- } \\
\text { Ratio } \\
\end{array}$ & $\begin{array}{l}\text { Mean } \\
\text { Squar } \\
\text { e } \\
\text { Error } \\
\end{array}$ & $\begin{array}{c}\text { De- } \\
\text { noising }\end{array}$ & $\begin{array}{l}\text { Peak- } \\
\text { Signal- } \\
\text { Noise- } \\
\text { Ratio } \\
\end{array}$ & $\begin{array}{l}\text { Mean } \\
\text { Square } \\
\text { Error }\end{array}$ & $\underset{\mathrm{g}}{\text { Blurrin }}$ & Filtering & $\begin{array}{c}\text { Peak- } \\
\text { Signal- } \\
\text { Noise- } \\
\text { Ratio } \\
\end{array}$ & $\begin{array}{l}\text { Mean } \\
\text { Squar } \\
\text { e } \\
\text { Error }\end{array}$ & $\begin{array}{l}\text { Enhance } \\
\text { Image }\end{array}$ & $\begin{array}{c}\text { Peak- } \\
\text { Signal- } \\
\text { Noise- } \\
\text { Ratio }\end{array}$ & $\begin{array}{l}\text { Mean } \\
\text { Squar } \\
\mathrm{e} \\
\text { Error }\end{array}$ \\
\hline 1 & & 61.6432 & 0.04 & & 58.6308 & 0.09 & & & 63.6357 & 0.02 & & 71.4381 & 0.02 \\
\hline 2 & & $\begin{array}{l}60.401 \\
1\end{array}$ & 0.06 & & 58.1525 & 0.10 & & & 64.5243 & 0.02 & & 74.7456 & 0.01 \\
\hline 3 & & 58.5430 & 0.09 & & 57.6482 & $\begin{array}{r}0.1 \\
1\end{array}$ & & & 63.6130 & 0.02 & & 72.5483 & 0.01 \\
\hline 4 & & 61.3019 & 0.05 & & 59.6842 & 0.07 & & & 64.1777 & 0.03 & & 72.3048 & 0.02 \\
\hline 5 & & 58.2473 & 0.10 & & $\begin{array}{c}58.113 \\
4\end{array}$ & 0.13 & & & $\begin{array}{c}65.112 \\
3\end{array}$ & 0.04 & & 73.3345 & 0.01 \\
\hline
\end{tabular}

(A)

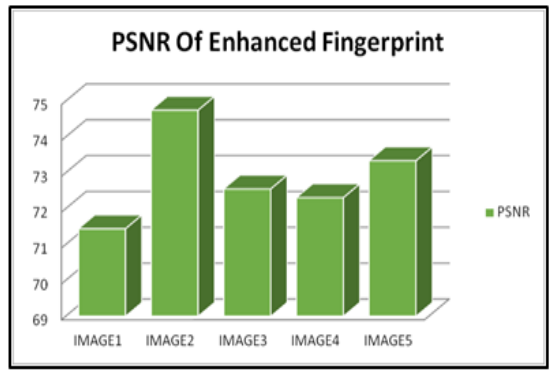

(B)

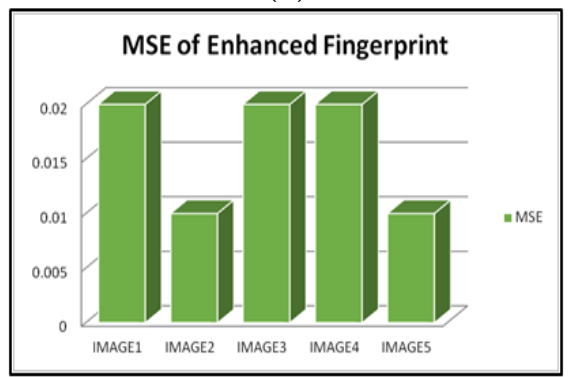

Fig. 10: (A) Graphical Representation for PSNR Values of Enhanced Fingerprint after the Removal of Gaussian Noise. (B) Graphical Representation for MSE Values of Enhanced Fingerprint after the Removal of Gaussian Noise

Table 2. Signifies the input image that undergone Pre-processing steps which include adding of Gaussian noise, blurring, filtering, and enhancement. The Table 2 also represents the performance measures namely Peak-signal-noise-ratio and Mean-Squared-Error after each iteration
Table 3 Signifies the input image that undergone Pre-processing steps which include adding of Speckle noise, blurring, filtering, and enhancement. The Table 3 also represents the performance measures namely Peak-signal-noise-ratio and Mean-Squared-Error after each iteration.

\subsection{Adding speckle noise}


Table 3: Enhanced Fingerprint Images with Its PSNR and MSE

\begin{tabular}{|c|c|c|c|c|c|c|c|c|c|c|c|c|c|}
\hline S.No & $\begin{array}{l}\text { Input } \\
\text { Image }\end{array}$ & $\begin{array}{c}\text { Peak - } \\
\text { Signal - } \\
\text { Noise - } \\
\text { Ratio }\end{array}$ & $\begin{array}{c}\text { Mean } \\
\text { Squar } \\
\mathrm{e} \\
\text { Error }\end{array}$ & $\begin{array}{c}\text { De- } \\
\text { noising }\end{array}$ & $\begin{array}{c}\text { Peak - } \\
\text { Signal - } \\
\text { Noise - } \\
\text { Ratio }\end{array}$ & $\begin{array}{l}\text { Mean } \\
\text { Squar } \\
\text { e } \\
\text { Error }\end{array}$ & Blurring & Filtering & $\begin{array}{c}\text { Peak - } \\
\text { Signal - } \\
\text { Noise- } \\
\text { Ratio }\end{array}$ & $\begin{array}{l}\text { Mean } \\
\text { Squar } \\
\text { e } \\
\text { Error }\end{array}$ & $\begin{array}{l}\text { Enhance } \\
\text { Image }\end{array}$ & $\begin{array}{c}\text { Peak - } \\
\text { Signal - } \\
\text { Noise - } \\
\text { Ratio }\end{array}$ & $\begin{array}{c}\text { Mean } \\
\text { Square } \\
\text { Error }\end{array}$ \\
\hline 1 & & 61.6432 & 0.04 & & 57.7560 & 0.11 & & & 65.3567 & 0.04 & & 73.4381 & 0.02 \\
\hline 2 & & 60.4011 & 0.06 & & 56.7182 & 0.10 & & & 67.2461 & 0.02 & & 75.7456 & 0.01 \\
\hline 3 & & 58.5430 & 0.09 & & 57.2455 & 0.12 & & & 65.6130 & 0.02 & & 73.5483 & 0.02 \\
\hline 4 & & 61.3019 & 0.05 & & 58.6623 & 0.09 & & & 64.1777 & 0.03 & & 73.3048 & 0.02 \\
\hline 5 & & 58.2473 & 0.10 & & 56.3999 & 0.15 & & & 69.1123 & 0.03 & & 76.3345 & 0.01 \\
\hline
\end{tabular}

(A)

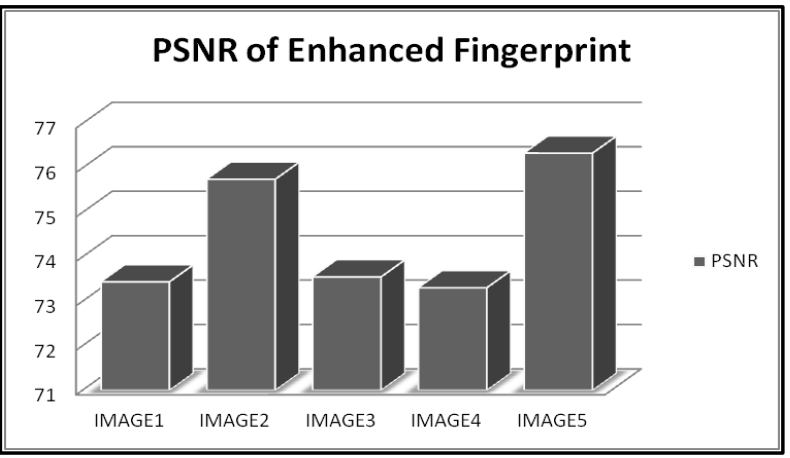

(B)

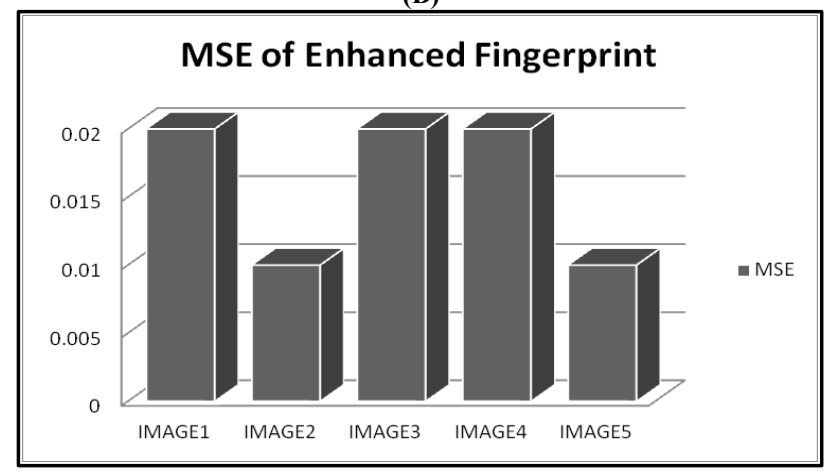

Fig. 11: (A) Graphical Representation for PSNR Values of Enhanced Fingerprint after the Removal of Speckle Noise. (B) Graphical Representation for MSE Values of Enhanced Fingerprint after the Removal of Speckle Noise.

\section{Comparative study of the proposed meth- odology with existing methodologies}

Table 4: Comparison of Proposed Methodology with Existing Methodologies

\begin{tabular}{ccccc}
\hline Author Name & $\begin{array}{c}\text { Subba } \\
\text { Reddy } \\
\text { Borra et al }\end{array}$ & $\begin{array}{c}\text { Subba } \\
\text { Reddy } \\
\text { Borra et al }\end{array}$ & $\begin{array}{c}\text { S.Neethu } \\
\text { et al }\end{array}$ & $\begin{array}{c}\text { Proposed } \\
\text { E nhancement } \\
\text { Method }\end{array}$ \\
\hline Technique & $\begin{array}{c}\text { Modified } \\
\text { Cuckoo } \\
\text { Search }\end{array}$ & $\begin{array}{c}\text { Cuckoo } \\
\text { Search }\end{array}$ & $\begin{array}{c}\text { Fourier } \\
\text { Transform }\end{array}$ & Wiener \\
PSNR & 66.6275 & 58.3672 & 53.5141 & 73.1627 \\
\hline
\end{tabular}

Table 4 given above presents the comparative study of the proposed enhancement methodology with other existing methodology in the literature. The PSNR values for proposed and existing methods are evaluated and given in the table 4.

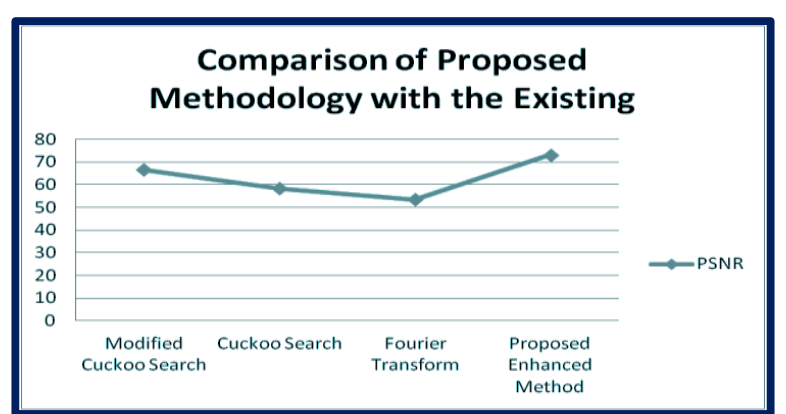

Fig. 12: Graphical Representation of Comparison Chart of Proposed Methodology.

Fig 12 gives the pictorial representation of the comparison chart of proposed methodology with other existing methods based on the PSNR values and techniques used. From the graph, it is apparent that our proposed method provides a better result when compared with the existing methods

\section{Conclusion}

An Efficient Fingerprint enhancement methodology has been defined. The proposed method uses Wiener Filter with re-blurring followed by three enhancement operations. The proposed methodology consists of all the possible enhancement operations. The 
step by step illustration of the proposed method was also stated in this paper. The experimental observation of the methodology has been predicted by adding three different noises such as salt and pepper, Gaussian noise and speckle noise and the performance was measured by two parameter measures namely PSNR and MSE .The values were tabulated and picturized separately. The paper also focused on the comparative study of proposed methodology than with other existing methodologies. The result shows the effectiveness of the proposed methodology as it gives a gives a better PSNR value of 73.1627 when compared with other existing methods.

\section{References}

[1] Neethu S, Sreelakshmi S \& Sankar D, "Enhancement of fingerprint using FFT $\times \mid$ FFT $\mid n$ filter", Procedia Computer Science, Vol.46, pp. 1561-1568, (2015). https://doi.org/10.1016/i.procs.2015.02.083.

[2] Gnanasivam P \& Muttan S, "An efficient algorithm for fingerprint preprocessing and feature extraction", Procedia Computer Science, Vol.2, No.2009, pp.133-142, (2010).

[3] Borra SR, Reddy GJ \& Reddy ES, “An Efficient Fingerprint Enhancement Technique Using Wave Atom Transform and MCS Algorithm", Procedia Computer Science, Vol.89, pp.785-793, (2016). https://doi.org/10.1016/j.procs.2016.06.061.

[4] Zhao F \& Tang X, "Preprocessing and postprocessing for skeletonbased fingerprint minutiae extraction", Pattern Recognition, Vol.40, No.4, pp.1270-1281,

(2007). https://doi.org/10.1016/j.patcog.2006.09.008.

[5] Chaurasia OP, "An Approach to Fingerprint Image Pre-Processing", International Journal of Image, Graphics and Signal Processing, Vol.4, No.6, pp.29-35, https://doi.org/10.5815/ijigsp.2012.06.05.

[6] Selia IG \& Parthiban L, "Approaches for Enhancing Fingerprint Images Using Filters: A Case Study", ACS-International Journal in Computational Intelligence, Vol.2, No.1, pp.1-9, (2011).

[7] Rajkumar R \& Hemachandran K, "A Review on Image enhancement of fingerprint using Directional filters", Assam University Journal of Science and Technology, Vol.7, No.2, pp.52-57, (2011).

[8] Greenberg S, Aladjem M \& Kogan D, "Fingerprint Image Enhancement using Filtering Techniques", Real-Time Imaging, Vol.8, No. 3, pp.227-236, (2002). https://doi.org/10.1006/rtim.2001.0283.

[9] Kausalya R \& Ramya A, "Latent Fingerprint Image Enhancemen Techniques", International Journal of Advanced Research in Computer and Communication Engineering, Vol.3, No.2, pp.5671-5674, (2014).

[10] Nagendra G, "Fingerprint Image Enhancement Using Filtering", International Journal of Computer Science Engineering, Vol.1, No.1, pp.61-78, (2012).

[11] Mather P \& Koch M, "Image Enhancement Techniques", Processing of Remotely-Sensed Images:An, Vol.1, No.3, pp.215-217, (2012).

[12] http://www.ni.com/white-paper/13306/en/. 